\title{
Research Article \\ On the Integrability of the SIR Epidemic Model with Vital Dynamics
}

\author{
Ding Chen \\ School of Economics and Management, Xi'an Shiyou University, Xi'an, China \\ Correspondence should be addressed to Ding Chen; chend@xsyu.edu.cn
}

Received 28 February 2020; Revised 20 May 2020; Accepted 21 May 2020; Published 6 July 2020

Academic Editor: Marin Marin

Copyright (C) 2020 Ding Chen. This is an open access article distributed under the Creative Commons Attribution License, which permits unrestricted use, distribution, and reproduction in any medium, provided the original work is properly cited.

\begin{abstract}
In this paper, we study the SIR epidemic model with vital dynamics $\dot{S}=-\beta S I+\mu(N-S), \dot{I}=\beta S I-(\gamma+\mu) I, \dot{R}=\gamma I-\mu R$, from the point of view of integrability. In the case of the death/birth rate $\mu=0$, the SIR model is integrable, and we provide its general solutions by implicit functions, two Lax formulations and infinitely many Hamilton-Poisson realizations. In the case of $\mu \neq 0$, we prove that the SIR model has no polynomial or proper rational first integrals by studying the invariant algebraic surfaces. Moreover, although the SIR model with $\mu \neq 0$ is not integrable and we cannot get its exact solution, based on the existence of an invariant algebraic surface, we give the global dynamics of the SIR model with $\mu \neq 0$.
\end{abstract}

\section{Introduction and Statement of the Main Results}

Over the past one hundred years, the mathematical modelling of epidemics has been the object of a large number of studies. The Susceptible-Infected-Recovered (SIR) model is one of the most interesting and best understood nonlinear epidemic models $[1,2]$. The first SIR model was proposed by Kermack and McKendrick in 1927 [2]. After that, many different epidemic models including time delay, age structure, space factor, white noise, multigroup, and seasonality have been proposed and studied (see [1] and the references therein). Observing the spread of marketing message is analogous to an epidemic, Rodrigues and Fonseca [3] used a SIR model to study the effects of a viral marketing strategy.

We consider the SIR epidemic model with vital dynamics which is given by

$$
\begin{aligned}
& \dot{S}=-\beta S I+\mu(N-S), \\
& I=\beta S I-(\gamma+\mu) I, \\
& \dot{R}=\gamma I-\mu R,
\end{aligned}
$$

where $S$ is the number of healthy individuals who are susceptible to the disease, $I$ is the number of infected individuals who can transmit the disease to the healthy ones, and $R$ is the number of individuals who have been infected and then recovered from the disease and the parameters $\beta, \gamma, \mu$, and $N$ denote the average number of contacts per infective per day, the recovery rate, the death rate, and the initial total fixed number of the individuals, respectively. It is assumed that the birth rate is equal to the death rate in this model (1). For $\mu=0$, it is called the SIR model without vital dynamics and was proposed by Kermack and McKendrick [2].

The aim of this paper is to study system (1) from the integrability point of view. The integrability of differential equations has been an old and important problem and has attracted much attention. Many scholars have developed a lot of ways to deal with the integrability for both partial differential equations (see [4-13] for instance) and ordinary differential equations (see [14-16] for instance). In particular, the existence of first integrals plays a crucial role in the integrability of ordinary differential equations [17-20]. If the considered system of ordinary differential equations admits a straight line solution, by the differential Galois method, one can usually prove that this system has no rational first 
integrals for almost all the parameters [21-23]. However, this method cannot tell whether this system is integrable for the remaining parameters. The SIR epidemic model (1) is exactly the case. Another tool that deals with the integrability of $3 \mathrm{D}$ differential systems is the Darboux theory of integrability $[15,16]$, which is a useful tool to find first integrals for polynomial ordinary differential equations, and has been successfully applied in many nonlinear models [24-27]. This theory can also help us make a more precise analysis of the global dynamics of a system topologically (see [28, 29] and the references therein). In the framework of the Darboux theory of integrability, we will give a complete classification of the irreducible Darboux polynomials, of the polynomial first integrals, of the proper rational first integrals, and of the algebraic integrability for the SIR model.

In the case of the constant population, i.e., $\mu=0$, the SIR model (1) admits a first integral and can be reduced into a planar system with respect to the variables $(S, I)$. The integrability of the reduced planar system has been investigated extensively by different methods, namely, the Adomian decomposition method [30], the homotopy analysis method [31], and the variational iteration method [32]. Recently, Bohner et al. investigated a rational SIR model with the constant population and the time-dependent coefficients and present an alternative solution method to Gleissner's approach [33]. Based on a quantum mechanical method, Williams et al. provided an exact analytical solution of the stochastic SIR model with the constant population [34]. However, in the case of the varying population, i.e., $\mu \neq 0$, the integrability of the full SIR model is an area where little research has been done. Harko et al. reduced the SIR model (1) into the Abel equation and provided its form series solution by using a perturbation approach [35]. To our knowledge, the first integrals of the SIR model (1) with $\mu \neq 0$ have not been investigated previously. The aim of this work is to cover this gap.

Recall that a real polynomial $F(S, I, R) \in \mathbb{R}[S, I, R]$ is called a Darboux polynomial of system (1) if it satisfies

$$
\begin{aligned}
(-\beta S I & +\mu(N-S)) \frac{\partial F}{\partial S}+(\beta S I-(\gamma+\mu) I) \frac{\partial F}{\partial I}+(\gamma I-\mu R) \frac{\partial F}{\partial R} \\
& =K F
\end{aligned}
$$

for some polynomial $K(S, I, R) \in \mathbb{R}[S, I, R]$, called a cofactor of $F$. Clearly, if $F$ is a Darboux polynomial, then $F=0$ is invariant with respect to the flow of system (1). Hence, we call $F=0$ an invariant algebraic surface of system (1). It is well known that a polynomial function $f=f_{1}^{n_{1}}, \cdots, f_{m}^{n_{m}}$ is a Darboux polynomial iff each irreducible factor $f_{i}, i=1, \cdots$, $m$ is also a Darboux polynomial. Hence, for simplicity, we only focus on the irreducible Darboux polynomials of system (1).

Our main result on the integrability of system (1) is as follows, which characterizes all irreducible Darboux polynomials of system (1).
Theorem 1. All irreducible Darboux polynomials of system (1) consist of $F_{1}(S, I, F)=S+I+R-N$ with the cofactors $K_{1}(S$, $I, R)=-\mu$ and $F_{2}(S, I, F)=I$ with the cofactor $K_{2}(S, I, R)=$ $\beta S-\gamma-\mu$.

In addition, Darboux polynomials can help us construct the algebraic (polynomial or rational) first integrals. By Theorem 1 , we can easily obtain the following result.

Corollary 2. The following statements hold for system (1).

(1) It has a polynomial first integral if and only if $\mu=0$, and in this case, the polynomial first integral is $F=S$ $+I+R$

(2) It has no any proper rational first integral

(3) It is not algebraically integrable

It is not surprising that the SIR model without vital dynamics, i.e., $\mu=0$, has a first integral $F=S+I+R$ which is the total number of individuals in the given population. Furthermore, system (1) with $\mu=0$ has another first integral $G(S, I, R)=\gamma \ln S+\beta R$, which can help us compute the number $S(+\infty)$ of individuals that will never contract the infection. Let us mention that the first integral $G=\gamma \ln S+\beta R$ can be built by the classical Jacobi last multiplier method, observing system (1) admits a Jacobi last multiplier $M=1 / S$ $I$. In a word, the system with $\mu=0$ is a completely integrable system with two functionally independent first integrals $F, G$. Based on this fact, we have the following remarks on its integrability.

(i) The orbits of system (1) with $\mu=0$ are contained in the curves

$$
\left\{(S, I, R) \mid F(S, I, R)=c_{1}, \quad G(S, I, R)=c_{2}\right\}
$$

and its general solutions are given by the following implicit functions:

$$
\begin{aligned}
& R=-\frac{\gamma}{\beta} \ln S+\frac{c_{2}}{\beta}, \\
& I=\frac{\gamma}{\beta} \ln S-S-\frac{c_{2}}{\beta}+c_{1}, \\
& \int \frac{1}{S\left(\beta S-\gamma \ln S+c_{2}-\beta c_{1}\right)} d S=t+c_{3},
\end{aligned}
$$

where $c_{1}, c_{2}, c_{3}$ are constants

(ii) System (1) with $\mu=0$ has two Lax formulations $\dot{L}_{i}$ $=\left[L_{i}, N_{i}\right], i=1,2$, where the matrices $L_{i}$ and $N_{i}$ are given by 


$$
\begin{aligned}
& L_{1}:=\left(\begin{array}{ccc}
0 & -2 \sqrt{R} & 2 \sqrt{I} \\
2 \sqrt{R} & 0 & -2 \sqrt{S} \\
-2 \sqrt{I} & 2 \sqrt{S} & 0
\end{array}\right), \\
& N_{1}:=\left(\begin{array}{ccc}
0 & -1 & \frac{\beta I \sqrt{S}+2 \sqrt{I}}{2 \sqrt{R}} \\
1 & 0 & -\frac{\beta S \sqrt{I}+2 \sqrt{S}-\gamma \sqrt{I}}{2 \sqrt{R}} \\
-\frac{\beta I \sqrt{S}+2 \sqrt{I}}{2 \sqrt{R}} & \frac{\beta S \sqrt{I}+2 \sqrt{S}-\gamma \sqrt{I}}{2 \sqrt{R}} & 0 \\
0 & -1 & i(1+S)+R \\
1 & 0 & -1-S+i R \\
-i(1+S)-R & 1+S-i R & 0 \\
0 & 0 & I(\beta S+i \gamma) \\
0 & 0 & I(i \beta S-\gamma) \\
-I(\beta S+i \gamma) & -I(i \beta S-\gamma) & 0
\end{array}\right), \\
& L_{2}:=\left(\begin{array}{ccc}
0 & 0
\end{array}\right)
\end{aligned}
$$

(iii) System (1) with $\mu=0$ has infinitely many HamiltonPoisson realizations parameterized by the group $S$ $L(2, \mathbb{R})$; that is, $\left(\mathbb{R}^{3},\{\cdot, \cdot\}_{a b}, H_{c d}\right)$ is a HamiltonPoisson realization where the Poisson bracket $\{\cdot, \cdot\}$ reads

$$
\{f, g\}_{a b}:=v \nabla C_{a b} \cdot(\nabla f \times \nabla g),
$$

for any functions $f, g \in C^{\infty}\left(\left(\mathbb{R}^{+}\right)^{3}, \mathbb{R}\right), v=1 /(S I)$, the Casimir function

$$
C_{a b}=a(\ln (S)+\beta R)+b(S+I+R)
$$

the Hamiltonian function

$$
H_{c d}=c(\ln (S)+\beta R)+d(S+I+R)
$$

and the coefficients $a, b, c, d \in \mathbb{R}$ such that $a d-b c=1$

As mentioned above, the SIR model with $\mu=0$ is integrable with two first integrals, which implies it is orbitally equivalent to a linear differential system. Meanwhile, the SIR model with $\mu \neq 0$ has no polynomial/rational first integrals. However, based on the existence of an invariant algebraic surface, we can characterize the global phase portraits of the SIR model with $\mu \neq 0$, which helps us understand the final evolutions of this model and the spread of the disease.

Theorem 3. The following statements hold for system (1) with $\mu \neq 0$.

(a) All orbits with initial points not on the invariant algebraic surface $F_{1}=0$ and not at infinity are heteroclinic ones, which all positively approach the surface $F_{1}=0$ and negatively go to infinity

(b) The dynamics of system (1) at the infinity $\mathbb{S}^{2}$ is topologically equivalent to the one described in Figure 1

(c) System (1) on the invariant algebraic surface $F_{1}=0$ has four topologically different phase portraits, which are described in Figure 2

Theorem 3 provides the final evolutions of this model. As we have shown, there are only two possible final evolutions for this model. In the first type, both infective and recovered individuals tend to zero; that is, the disease fails to spread, and in the second type, the infective individuals cannot tend to zero; that is, the disease is endemic.

The paper is organized as follows: in Section 2, we prove Theorem 1 and Corollary 2. The proof of Theorem 3 will be given in Section 3. In the last section we draw our conclusions, including some discussions on the biological meaning of our results.

\section{Proof of Theorem 1 and Corollary 2}

2.1. Proof of Theorem 1. When $\mu=0$, it is easy to see that $S+I+R-N$ is a Darboux polynomial of system (1) with zero cofactor. In what follows, we deal with the case $\mu \neq 0$. For simplicity, we introduce the change of variables

$$
\begin{aligned}
& S=\frac{\gamma+\mu}{\beta} x, \\
& I=\frac{\gamma+\mu}{\beta} y, \\
& R=\frac{\mu}{\beta} z
\end{aligned}
$$

and a time rescaling $t=\tilde{t} /(\mu+\gamma)$. Then, system (1) becomes

$$
\begin{aligned}
& x^{\prime}=a-b x-x y, \\
& y^{\prime}=-y+x y, \\
& z^{\prime}=y-b z,
\end{aligned}
$$

where the prime ' denotes a derivative with respect to the new time $\tilde{t}$ and the coefficients $a=\mu N \beta /(\mu+\gamma)^{2}>0$ and $b=\mu /(\mu+\gamma) \in(0,1)$. Clearly, to complete the proof of Theorem 1, we need only to prove the next result.

Proposition 4. All irreducible Darboux polynomials of system (10) consist of $f(x, y, z)=x+y+(1-b) z-a / b$ with the cofactors $k_{1}(x, y, z)=-b$ and $f_{2}(x, y, z)=y$ with the cofactor $k_{2}(x, y, z)=x-1$.

Suppose $f(x, y, z)$ is a Darboux polynomial of system (10) with the cofactor $k(x, y, z)$, that is,

$$
(a-b x-x y) \frac{\partial f}{\partial x}+(x y-y) \frac{\partial f}{\partial y}+(y-b z) \frac{\partial f}{\partial z}=k f
$$




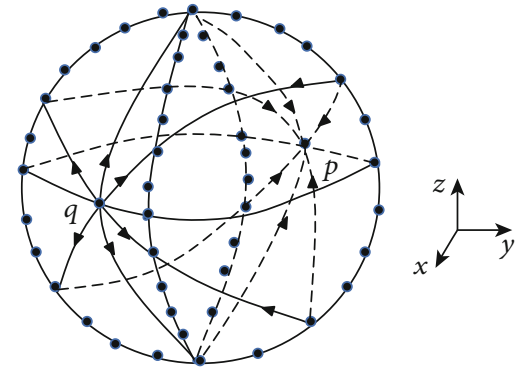

Figure 1: Phase portrait at infinity on the Poincaré ball of system (1). Note that system (1) has two closed curves of equilibria $\{x=0$, $\left.y^{2}+z^{2}=1\right\} \cup\left\{y=0, x^{2}+z^{2}=1\right\}$ and two nodes $q, p$.

Comparing the degree of both sides of (11), we get that the cofactor $k(x, y, z)$ is a polynomial with degree less than two. Without loss of generality, we set $k(x, y, z)=k_{0}+k_{1} x+$ $k_{2} y+k_{3} z$. Then, we claim $k_{3}=0$. In fact, we write $f$ as powers in the variable $z$, i.e., $f(x, y, z)=\sum_{j=0}^{n} f_{j}(x, y) z^{j}$, where each $f_{j}$ is a polynomial in the variables $x$ and $y$ and $f_{n} \neq 0$. Computing the coefficient in (11) of $z^{n+1}$, we have $0=k_{3} f_{n}$ $(x, y)$ which implies $k_{3}=0$.

We make the change of the variables $x=\alpha^{-1} X, y=\alpha^{-1} Y$, $z=Z$, and $\tilde{t}=\alpha T$ and rewrite (10) into

$$
\begin{aligned}
& \frac{d X}{d T}=-X Y-\alpha b X+\alpha^{2} a, \\
& \frac{d Y}{d T}=X Y-\alpha Y, \\
& \frac{d Z}{d T}=Y-\alpha b Z .
\end{aligned}
$$

Let $n$ be the highest weight degree in the weight homogeneous components of $f$ in $x, y, z$ with the weight exponent $(1,1,0)$. Set

$$
\begin{aligned}
F(X, Y, Z)= & \alpha^{n} f\left(\alpha^{-1} X, \alpha^{-1} Y, Z\right)=F_{0}(X, Y, Z) \\
& +\alpha F_{1}(X, Y, Z)+\cdots+\alpha^{n} F_{n}(X, Y, Z), \\
K(X, Y, Z)= & \alpha k\left(\alpha^{-1} X, \alpha^{-1} Y, Z\right)=k_{1} X+k_{2} Y+\alpha k_{0},
\end{aligned}
$$

where $F_{i}$ is a weight homogeneous polynomial of weight degree $(n-i)$ in $(X, Y, Z)$. Since $f$ is a Darboux polynomial of system (10) with the cofactor $k$, it is not difficult to check that $F$ is a Darboux polynomial of system (12) with the cofactor $K$, that is,

$$
\begin{aligned}
& \left(-x y-\alpha b x+\alpha^{2} a\right) \sum_{i=0}^{n} \alpha^{i} \frac{\partial F_{i}}{\partial x}+(x y-\alpha y) \sum_{i=0}^{n} \alpha^{i} \frac{\partial F_{i}}{\partial y} \\
& +(y-\alpha b z) \sum_{i=0}^{n} \alpha^{i} \frac{\partial F_{i}}{\partial z}=\left(k_{1} x+k_{2} y+\alpha k_{0}\right) \sum_{i=0}^{n} F_{i}
\end{aligned}
$$

where we still use $x, y, z$ instead of $X, Y, Z$.

Equating the terms with $\alpha^{0}$ in (14) yields

$$
L\left[F_{0}\right]=\left(k_{1} x+k_{2} y\right) F_{0}
$$

where $L$ stands for a linear partial differential operator

$$
L:=-x y \frac{\partial}{\partial x}+x y \frac{\partial}{\partial y}+y \frac{\partial}{\partial z} .
$$

To solve (15), we introduce the change of variables

$$
\begin{aligned}
& u=x, \\
& v=x+y \\
& w=x \exp (z)
\end{aligned}
$$

and transform (15) into

$$
u(u-v) \frac{\partial \bar{F}_{0}}{\partial u}=\left(\left(k_{1}-k_{2}\right) u+k_{2} v\right) \bar{F}_{0}
$$

where $\bar{F}_{0}$ is $F_{0}$ written in terms of $u, v, w$. Solving (18) yields $\bar{F}_{0}=G_{0}(v, w)(v-u)^{k_{1}} u^{-k_{2}}$ with $G_{0}$ being an arbitrary smooth function in $v, w$. Clearly, in order for $F_{0}(x, y, z)=$ $\bar{F}_{0}(u, v, w)=G_{0}(x+y, x \exp (z)) y^{k_{1}} x^{-k_{2}}$ to be a weight homogeneous polynomial of degree $n$ in $x, y, z$, we must have $k_{1}=m, k_{2}=-s$ and

$$
F_{0}=a_{0} x^{s} y^{m}(x+y)^{l}, \quad s+m+l=n,
$$

for some nonzero number $c_{0} \in \mathbb{R} /\{0\}$ and nonnegative integers $s, m, l \in \mathbb{N} \cup\{0\}$.

Similarly, equating the terms with $\alpha$ in (14) leads to

$$
L\left[F_{1}\right]=m x F_{1}-s y F_{1}+b x \partial_{x} F_{0}+y \partial_{y} F_{0}+b z \partial_{z} F_{0}+k_{0} F_{0} .
$$

Substituting $F_{0}=a_{0} x^{s} y^{m}(x+y)^{l}$ into (20) yields

$$
\begin{aligned}
L\left[F_{1}\right]= & (m x-s y) F_{1}+a_{0} x^{s} y^{m}(x+y)^{1-1}\left\{\left(b s+b l+m+k_{0}\right) x\right. \\
& \left.+\left(b s+l+m+k_{0}\right) y\right\} .
\end{aligned}
$$

Proceeding as above, by (17) we rewrite (21) as

$$
\begin{aligned}
u(u-v) \frac{\partial \bar{F}_{1}}{\partial u}= & (m u+s(u-v)) \bar{F}_{1}+a_{0} v^{l-1} u^{s}(v-u)^{m}\{(b s+b l \\
& \left.\left.+m+k_{0}\right) u+\left(b s+l+m+k_{0}\right)(v-u)\right\} .
\end{aligned}
$$




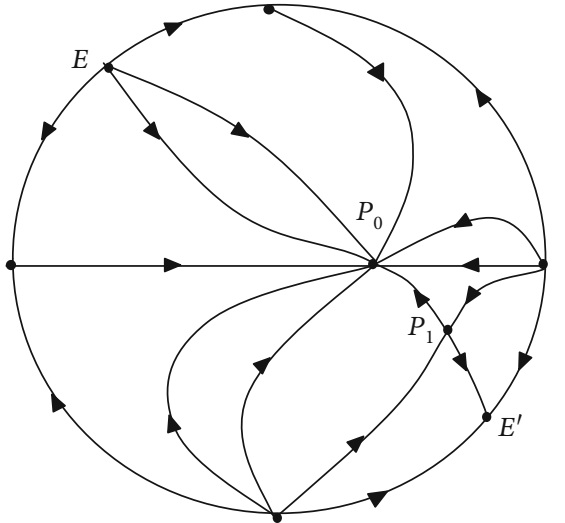

(a) $R_{0}<1$

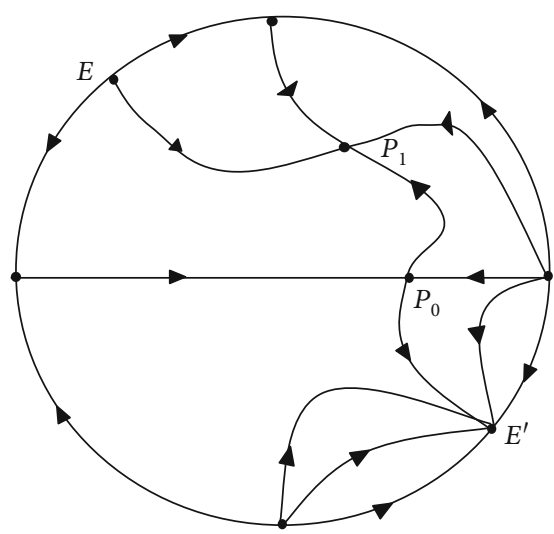

(c) $R_{0}>1, \Delta \geq 0$

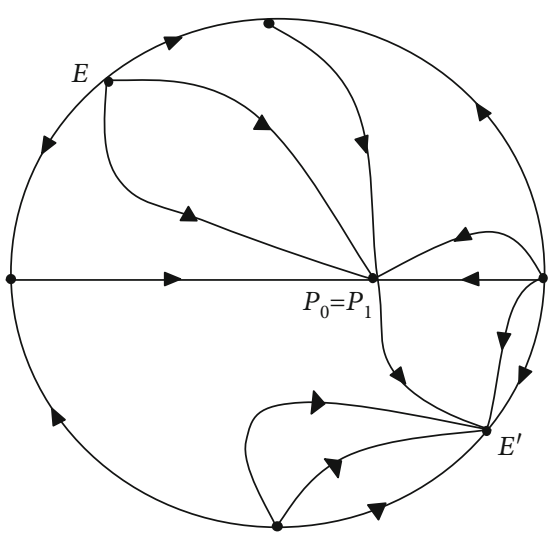

(b) $R_{0}=1$

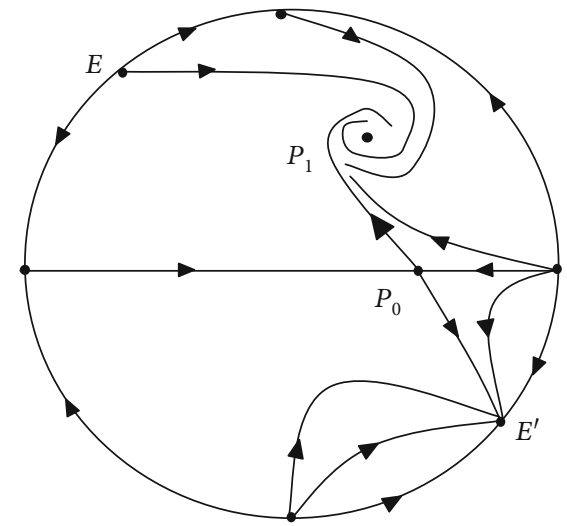

(d) $R_{0}>1, \Delta<0$

Figure 2: Phase portraits of system (1) on the invariant planes $F_{1}=0$.

Integrating the above equation with respect to $u$, we obtain

$$
\begin{aligned}
F_{1}= & \bar{F}_{1}=a_{0} u^{s}(v-u)^{m} v^{l-1}\left\{\left(b s+b l+m+k_{0}\right) \ln |u-v|\right. \\
& \left.+\left(b s+l+m+k_{0}\right) \ln \left|\frac{w}{u}\right|+G_{1}(v, w)\right\} \\
= & a_{0} x^{s} y^{m}(x+y)^{l-1}\left\{\left(b s+b l+m+k_{0}\right) \ln |y|\right. \\
& \left.+\left(b s+l+m+k_{0}\right) z+G_{1}(x+y, x \exp (z))\right\},
\end{aligned}
$$

with $G_{1}$ being an arbitrary smooth function. In order for $F_{1}$ to be a weight-homogeneous polynomial of weight degree $n-1$, we must have $G_{1}=c_{1} \in \mathbb{R}$ which is a constant, $k_{0}=$ $-b s-b l-m$, and

$$
F_{1}=a_{0} x^{s} y^{m}(x+y)^{l-1}\left(l(1-b) z+c_{1}\right)
$$
have

Equating the terms with $\alpha^{j}$ in (14) for $j=2,3, \cdots, n$, we

$$
\begin{aligned}
L\left[F_{j}\right]= & (m x-s y) F_{j}+b x \partial_{x} F_{j-1}+y \partial_{y} F_{j-1} \\
& +b z \partial_{z} F_{j-1}+k_{0} F_{j-1}-a \partial_{x} F_{j-2} .
\end{aligned}
$$

In particular, for $j=2$ we substitute $F_{0}, F_{1}$ into (25) and obtain

$$
\begin{aligned}
L\left[F_{2}\right]= & (m x-s y) F_{2}+a_{0} x^{s} y^{m}(x+y)^{l-2}\{(l(1-b) z \\
& \left.+c_{1}\right)(-b l(x+y)+b(l-1) x+(l-1) y) \\
& \left.+b l(1-b)(x+y) z-\frac{a s(x+y)^{2}}{x}-a l(x+y)\right\}
\end{aligned}
$$

or equivalently

$$
\begin{aligned}
u(u-v) \frac{\partial \bar{F}_{2}}{\partial u}= & (m u+s(u-v)) \bar{F}_{2} \\
& +a_{0} u^{s}(v-u)^{m} v^{l-2}\left\{-\ln \frac{w}{u}(1-b)^{2} l(l\right. \\
& -1)(u-v)-\left(b c_{1}+a l\right) u+\left(c_{1} b l+c_{1}\right. \\
& \left.\left.-c_{1} l+a l\right)(u-v)-\frac{a s v^{2}}{u}\right\} .
\end{aligned}
$$


Solving the above linear differential equation, we get

$$
\begin{aligned}
F_{2}= & \bar{F}_{2}=a_{0} x^{s} y^{m}(x+y)^{l-2}\left\{\frac{l(l-1)(1-b)^{2} z^{2}}{2}\right. \\
& -\left(b c_{1}+a l+a s\right) \ln |y|-\left(c_{1} b l+c_{1}-c_{1} l+a l+a s\right) z \\
& \left.-\frac{a s(x+y)}{x}+G_{1}(x+y, x \exp (z))\right\} .
\end{aligned}
$$

In order for $F_{2}$ to be a weight-homogeneous polynomial of weight degree $n-1$, we have $b c_{1}+a l+a s=0$, $G_{2}=c_{2} \in \mathbb{R}$ which implies

$$
\begin{aligned}
F_{1}= & a_{0} x^{s} y^{m}(x+y)^{l-1}\left(\left(\begin{array}{l}
l \\
1
\end{array}\right)(1-b) z-\left(\begin{array}{l}
l \\
1
\end{array}\right) \frac{a}{b}-\frac{a s}{b}\right), \\
F_{2}= & a_{0} x^{s} y^{m}(x+y)^{l-2}\left(\left(\begin{array}{l}
l \\
2
\end{array}\right)(1-b)^{2} z^{2}-\left(\begin{array}{l}
l \\
2
\end{array}\right) \frac{2 a(1-b) z}{b}\right. \\
& \left.+a s\left(\frac{(l-1)(b-1) z}{b}-\frac{x+y}{x}\right)+c_{2}\right) .
\end{aligned}
$$

Proceeding as above, from (25) with $j=3$ and (29), we get

$$
\begin{aligned}
L\left[F_{3}\right]= & (m x-s y) F_{3}+a_{0} x^{s} y^{m}(x+y)^{l-3}\{b z(x+y)(l(l-1)(1 \\
& \left.-b)^{2} z-\frac{a(l+s)(l-1)(1-b)}{b}\right)+\left(\frac{l(l-1)(1-b)^{2} z^{2}}{2}\right. \\
& \left.+\frac{a(l+s)(l-1)(b-1) z}{b}+c_{2}\right)(-2 b x-(b l+2-l) y) \\
& +a s\left(2 b+2 m+\frac{a(l+s)}{b}+l-1\right) \frac{(x+y)^{2}}{x} \\
& +\left(\frac{a^{2}(l-1)(l+s)}{b}-a s(l-1)\right)(x+y) \\
& \left.-\frac{a s(x+y)^{2} l(1-b) z}{x}-a l(l-1)(x+y)(1-b) z\right\} .
\end{aligned}
$$

Working in a similar way to solve $F_{2}$, we can prove that

$$
\begin{aligned}
F_{3}= & a_{0} x^{s} y^{m}(x+y)^{l-3}\left\{M_{1} z^{3}+M_{2} z^{2}+\left(M_{3}+M_{4} x+M_{4} y\right) z\right. \\
& +\left(M_{5}+M_{6} z\right) \ln |y|+M_{7} \operatorname{dil} \log \left(-\frac{y}{x}\right)+M_{8} \frac{x+y}{x} \\
& \left.+G_{3}(x+y, x \exp (z))\right\},
\end{aligned}
$$

where $\operatorname{dilog}(\cdot)$ is the dilogarithm function defined by

$$
\operatorname{dilog}(x)=\int_{1}^{x} \frac{\ln (t)}{1-t} d t
$$

and the coefficients $M_{i}, i=1, \cdots, 8$ are given by

$M_{1}=\left(\begin{array}{l}l \\ 3\end{array}\right)(1-b)^{3}$,

$M_{2}=-\left(\begin{array}{l}l \\ 3\end{array}\right) \frac{3 a(1-b)^{2}}{b}-a s(1-b)(2 l-1)$,

$M_{3}=a s\left(2 b+2 m+\frac{a(2 l-1+s)}{b}\right)-\left(c_{2} b l+2 c_{2}-c_{2} l-\frac{a^{2} l(l-1)}{b}\right)$,

$M_{4}=a s(1-b) l$,

$M_{5}=\frac{a^{2} l(l-1)}{b}-2 b c_{2}+a s\left(\frac{a(2 l+s-1)}{b}+2 b+2 m\right)$,

$M_{6}=-a s(1-b)(2 l-1)$,

$M_{7}=-a s(1-b)(2 l-1)$,

$M_{8}=a s\left(2 b+2 m+\frac{a(l+s)}{b}+2 l-1-b l\right)$.

In order for $F_{3}$ to be a weight-homogeneous polynomial of weight degree $n-3$, we have $M_{5}=M_{6}=M_{7}=0$ and the function $G_{3}$ is a constant, which implies

$$
\begin{aligned}
s= & , \\
c_{2}= & \left(\begin{array}{l}
l \\
2
\end{array}\right) \frac{a^{2}}{b^{2}}, \\
F_{1}= & a_{0} y^{m}(x+y)^{l-1}\left(\begin{array}{l}
l \\
1
\end{array}\right)\left((1-b) z-\frac{a}{b}\right), \\
F_{2}= & a_{0} y^{m}(x+y)^{l-2}\left(\begin{array}{l}
l \\
2
\end{array}\right)\left((1-b) z-\frac{a}{b}\right)^{2}, \\
F_{3}= & a_{0} y^{m}(x+y)^{l-3}\left(\begin{array}{l}
l \\
3
\end{array}\right)\left((1-b)^{3} z^{3}-\frac{3 a(1-b)^{2} z^{2}}{b}\right. \\
& \left.+\frac{3 a^{2}(1-b) z}{b^{2}}+c_{3}\right)
\end{aligned}
$$

with $c_{3}$ being a constant. Using the same argument similar to that above, we solve $F_{4}$ and get

$$
c_{3}=-\left(\frac{a}{b}\right)^{3}
$$




$$
\begin{aligned}
F_{4}= & a_{0} y^{m}(x+y)^{l-4}\left(\begin{array}{l}
l \\
4
\end{array}\right)\left((1-b)^{4} z^{4}-\frac{4 a(1-b)^{3} z^{3}}{b}\right. \\
& \left.+\frac{6 a^{2}(1-b)^{2} z^{2}}{b^{2}}-\frac{4 a^{3}(1-b) z}{b^{3}}+c_{4}\right)
\end{aligned}
$$

with $c_{4} \in \mathbb{R}$. Furthermore, by recursive calculations, we can prove that

$$
F_{j}=a_{0} y^{m}(x+y)^{l-j}\left(\begin{array}{l}
l \\
j
\end{array}\right)\left((1-b) z-\frac{a}{b}\right)^{j}, \quad j=0, \cdots, n .
$$

Therefore, the Darboux polynomial of system (10)

$$
\begin{aligned}
f & =\left.F\right|_{\alpha=1}=\sum_{j=0}^{n} a_{0}\left(\begin{array}{l}
l \\
j
\end{array}\right) y^{m}(x+y)^{l-j}\left((1-b) z-\frac{a}{b}\right)^{j} \\
& =a_{0} y^{m}\left((1-b) z-\frac{a}{b}\right)^{l}
\end{aligned}
$$

and the cofactor $k=\left.K\right|_{\alpha=1}=m x-(b+m)$, which completes the proof.

\subsection{Proof of Corollary 2}

2.2.1. Proof of Statement (1) of Corollary 2. It follows from Theorem 1 and the fact that a function $f$ is a polynomial first integral if and only if it is a Darboux polynomial with the zero cofactor.

2.2.2. Proof of Statement (2) of Corollary 2. Assume system (1) has a proper rational first integral $f=P / Q$ with $P, Q$ being relative prime; then, $P, Q$ are two different Darboux polynomials with the same nonzero cofactor; see [16] for instance, which contradicts Theorem 1 .

2.2.3. Proof of Statement (3) of Corollary 2. It follows from statements (1) and (2) and a well-known result that a polynomial vector field in $\mathbb{R}^{n}$ is algebraically integrable if and only if it has $n-1$ functionally independent rational first integrals.

\section{Proof of Theorem 3}

3.1. Proof of Statement (a) of Theorem 3. According to Theorem 1 , system (1) has a Darboux polynomial $F_{1}=S+I+$ $R-N$ with the cofactor $-\mu<0$, which implies that $I(S, I$, $R, t)=(S+I+R-N) \exp (\mu t)$ is a time-dependent first integral of system (1), that is, $d I / d t=0$ along the flow of (1). Let $\phi(t)=(S(t), I(t), R(t))$ be an arbitrary orbit of system (1) with the initial value $\phi(0)=\left(S_{0}, I_{0}, R_{0}\right)$ satisfying $F_{1}\left(S_{0}, I_{0}, R_{0}\right) \neq 0$. By

$$
(S(t)+I(t)+R(t)-N) \exp (\mu t)=S_{0}+I_{0}+R_{0-N} \in \mathbb{R} /\{0\},
$$

we see that $\phi(t)$ will approach the invariant surface $F_{1}=0$ as $t \longrightarrow+\infty$, whereas it goes to infinity as $t \longrightarrow-\infty$. Statement (a) follows.

3.2. Proof of Statement (b) of Theorem 3. In order to understand the global dynamics of system (1) at infinity, we will use the Poincaré compactification in $\mathbb{R}^{3}$ and analyze the flow at infinity for the local charts $U_{i}$ and $V_{i}, i=1,2,3$. See [36] for more details on the Poincaré compactification of a polynomial vector field.

3.2.1. In the Local Chart $U_{1}$ and $V_{1}$. Making the change of variables $(S, I, R)=\left(z_{3}^{-1}, z_{1} z_{3}^{-1}, z_{2} z_{3}^{-1}\right)$ and the time rescaling $d \tau=z_{3}^{-1} d t$, we obtain the Poincaré compactification of system (1) in the local chart $U_{1}$

$$
\begin{aligned}
& \frac{d z_{1}}{d \tau}=-z_{1}\left(N \mu z_{3}^{2}-\beta z_{1}+\gamma z_{3}-\beta\right), \\
& \frac{d z_{2}}{d \tau}=-N \mu z_{2} z_{3}^{2}+\beta z_{1} z_{2}+\gamma z_{1} z_{3}, \\
& \frac{d z_{3}}{d \tau}=-z_{3}\left(N \mu z_{3}^{2}-\beta z_{1}-\mu z_{3}\right) .
\end{aligned}
$$

The plane $z_{3}=0$ at infinity is invariant, which corresponds to the points on the sphere at infinity, and so system (1) restricted to $z_{3}=0$ becomes

$$
\begin{aligned}
& \frac{d z_{1}}{d \tau}=\beta z_{1}\left(z_{1}+1\right), \\
& \frac{d z_{2}}{d \tau}=\beta z_{1} z_{2},
\end{aligned}
$$

which has a line of singular points $z_{1}=0$ and an isolated singular point $\left(z_{1}, z_{2}\right)=(-1,0)$. Moreover, system (40) has a first integral $\Phi=\left(z_{1}+1\right) / z_{2}$. Using this first integral, the phase portrait on the local chart $U_{1}$ is described in Figure 3. The flow on the local chart $V_{1}$ is the same with the flow of $U_{1}$ by reversing the time since the compactified vector field in $V_{1}$ coincides with the vector field in $U_{1}$ multiplied by $-1[36]$.

3.2.2. In the Local Chart $U_{2}$ and $V_{2}$. To get the Poincaré compactification of system (1) in the local chart $U_{2}$, we take the change of variables $(S, I, R)=\left(z_{1} z_{3}^{-1}, z_{3}^{-1}, z_{2} z_{3}^{-1}\right)$ and the time rescaling $d \tau=z_{3}^{-1} d t$ and transform (1) into

$$
\begin{aligned}
& \frac{d z_{1}}{d \tau}=N \mu z_{3}^{2}-\beta z_{1}^{2}+\gamma z_{1} z_{3}-\beta z_{1}, \\
& \frac{d z_{2}}{d \tau}=-\beta z_{1} z_{2}+\gamma z_{2} z_{3}+\gamma z_{3}, \\
& \frac{d z_{3}}{d \tau}=z_{3}\left(-\beta z_{1}+\gamma z_{3}+\mu z_{3}\right) .
\end{aligned}
$$




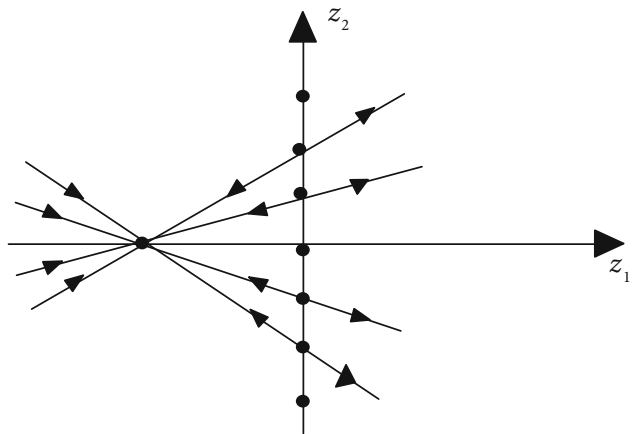

FIgURE 3: The phase portrait in the local chart $U_{1}$ at infinity.

When $z_{3}=0$, system (41) becomes

$$
\begin{aligned}
& \frac{d z_{1}}{d \tau}=-\beta z_{1}\left(z_{1}+1\right), \\
& \frac{d z_{2}}{d \tau}=-\beta z_{1} z_{2} .
\end{aligned}
$$

Clearly, system (42) coincides with system (40) by reversing the time. Then, the phase portrait on the local chart $U_{1}$ is described in Figure 4. Again, the flow in the local chart $V_{2}$ is the same as the flow in the local chart $U_{2}$ by reversing the time.

3.2.3. In the Local Chart $U_{3}$ and $V_{3}$. Proceeding as above, making the change of variables $(S, I, R)=\left(z_{1} z_{3}^{-1}, z_{2} z_{3}^{-1}, z_{3}^{-1}\right)$ and the time rescaling $d \tau=z_{3}^{-1} d t$, system (1) becomes

$$
\begin{aligned}
& \frac{d z_{1}}{d \tau}=N \mu z_{3}^{2}-\gamma z_{1} z_{2} z_{3}-\beta z_{1} z_{2}, \\
& \frac{d z_{2}}{d \tau}=-z_{2}\left(\gamma z_{2} z_{3}-\beta z_{1}+\gamma z_{3}\right), \\
& \frac{d z_{3}}{d \tau}=-z_{3}^{2}\left(\gamma z_{2}-\mu\right) .
\end{aligned}
$$

On the invariant plane $z_{3}=0$, system (43) is reduced to

$$
\begin{aligned}
& \frac{d z_{1}}{d \tau}=-\beta z_{1} z_{2}, \\
& \frac{d z_{2}}{d \tau}=\beta z_{1} z_{2} .
\end{aligned}
$$

System (44) has two lines of singular points $z_{1}=0$ and $z_{2}=0$. It also has a first integral $\Phi=z_{1}+z_{2}$, which helps us get its phase portrait as shown in Figure 5. The flow at infinity in the local chart $V_{3}$ is the same as the flow on the local chart $U_{3}$ by reversing the time.

To summarize the above analysis, one obtains a global picture of the dynamical behavior of system (1) on sphere $\mathbb{S}^{2}$ at infinity: it has two closed curves of equilibria $\{x=0$, $\left.y^{2}+z^{2}=1\right\} \cup\left\{y=0, x^{2}+z^{2}=1\right\}$ and two nodes (see Figure 1).

3.3. Proof of Statement (c) of Theorem 3. System (1) has the invariant algebraic surface $\{(S, I, R): S+I+R-N=0\}$.

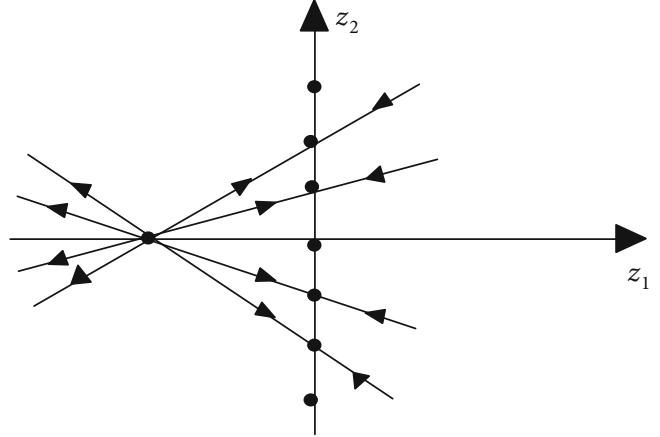

Figure 4: The phase portrait in the local chart $U_{2}$ at infinity.

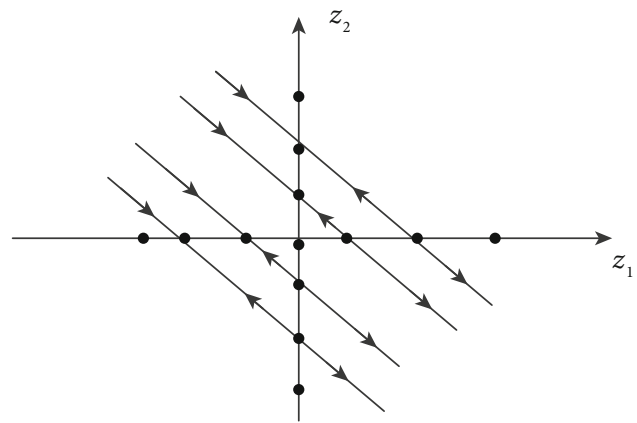

FIGURE 5: The phase portrait in the local chart $U_{3}$ at infinity.

Restricted to this invariant algebraic surface, system (1) becomes

$$
\begin{aligned}
& \dot{S}=-\beta S I+\mu(N-S):=P(S, I), \\
& \dot{I}=\beta S I-(\gamma+\mu) I:=Q(S, I) .
\end{aligned}
$$

We first study the finite singular points of system (45). Clearly, system (45) has two singular points

$$
\begin{aligned}
& P_{0}=(N, 0), \\
& P_{1}=\left(\frac{\gamma+\mu}{\beta}, \frac{\mu(N \beta-\gamma-\mu)}{\beta(\gamma+\mu)}\right) .
\end{aligned}
$$

One can check that the eigenvalues of the linear part at $P_{0}$ are $-\mu$ and $N \beta-\gamma-\mu$. Set $R_{0}=N \beta /(\gamma+\mu)$, called the basic reproduction number. If $R_{0}<1, P_{0}$ is a stable node. If $R_{0}>$ $1, P_{0}$ is a saddle. The eigenvalues of the linear part at $P_{1}$ are

$$
\begin{aligned}
& \frac{-\mu \beta N+\sqrt{\Delta}}{2(\mu+\gamma)}, \\
& \frac{-\mu \beta N-\sqrt{\Delta}}{2(\mu+\gamma)},
\end{aligned}
$$

with $\Delta=\left(\mu \beta N-2(\gamma+\mu)^{2}\right)^{2}-4 \gamma(\gamma+\mu)^{3}$. If $R_{0}<1, P_{1}$ is a saddle. If $R_{0}>1$ and $\Delta<0, P_{1}$ is a stable focus. If $R_{0}>1$ and $\Delta \geq 0, P_{1}$ is a stable node. In particular, if $R_{0}=1, P_{1}$ coincides with $P_{0}$, which is a semihyperbolic saddle-node point. 
Next, we turn to study the infinite singularities by the Poincaré compactification in $\mathbb{R}^{2}$. In the local chart $U_{1}$, where $S=z_{2}^{-1}, I=z_{1} z_{2}^{-1}$, and $d \tau=z_{2}^{-1} d t$, we have

$$
\begin{aligned}
& \frac{d z_{1}}{d \tau}=z_{1}\left(\beta-\gamma z_{2}+\beta z_{1}-N \mu z_{2}^{2}\right), \\
& \frac{d z_{2}}{d \tau}=z_{2}\left(\beta z_{1}+\mu z_{2}-N \mu z_{2}^{2}\right) .
\end{aligned}
$$

It has two singularities $(0,0)$ and $(-1,0)$ on the invariant line $z_{2}=0$. The eigenvalues of the linear part at $(-1,0)$ are $-\beta$ and $-\beta$, which implies this point is a stable node. By Theorem 2.19 in [37], we see that $(0,0)$ is a saddle-node point consisting of two hyperbolic sectors with one parabolic sector. Similarly, we take the change of variables $S=z_{1} z_{2}^{-1}$ and $I=$ $z_{2}^{-1}$ and the time rescaling $d \tau=z_{2}^{-1} d t$ to obtain the Poincaré compactification of system (45) in the local chart $U_{2}$

$$
\frac{d z_{1}}{d \tau}=N \mu z_{2}^{2}-\beta z_{1}^{2}+\gamma z_{1} z_{2}-\beta z_{1} \frac{d z_{2}}{d \tau}=z_{2}\left(\mu z_{2}-\beta z_{1}+\gamma z_{2}\right) .
$$

This system has an unstable node $(-1,0)$ and a saddle node $(0,0)$.

Finally, we show that system (45) has no limit cycles in $\mathbb{R}^{2}$. Since $I=0$ is an invariant manifold, limit cycles (if exits) must be in $I>0$ or $I<0$. In the region $\{(S, I): I>0\}$, observing

$$
\frac{\partial(P / I)}{\partial S}+\frac{\partial(Q / I)}{\partial I}=-\beta-\frac{\mu}{I}<0,
$$

it follows from the Dulac Theorem that system (45) has no limit cycles. In the region $\{(S, I): I<0\}$, system (45) has either no singular points or a saddle, which implies system (45) has no limit cycles.

Summarizing the above analysis, we obtain that the dynamics of the system (1) on $F_{1}=0$ is topologically described by one of Figure 2 on the Poincaré disk.

\section{Conclusions}

In this paper, by studying the invariant algebraic surfaces, we show that $\mu=0$ is the only value of the parameters for which the SIR epidemic model is integrable, and in this case, we provide its general solutions by implicit functions, two Lax formulations, and infinitely many Hamilton-Poisson realizations. When $\mu \neq 0$, the SIR model has no any algebraic first integral and we cannot get its exact solutions. However, based on the existence of the invariant algebraic surfaces, we characterize the topological structure of orbits for the SIR epidemic model with $\mu \neq 0$. Moreover, if the SIR model has a positive death/birth rate $\mu$, the disease will ultimately approach either the disease-free steady state $P_{0}$ on $F_{1}=0$ or the endemic steady state $P_{1}$ on $F_{1}=0$, depending on the basic reproduction number $R_{0}$. In case the $R_{0}$ is small, saying less than one, the SIR model has no positive equilibrium point and the disease approaches the disease-free steady state $P_{0}$ on $F_{1}=0$, which implies the disease will always fail to spread. In case the $R_{0}$ is big, saying larger than one, the SIR model admits a new positive equilibrium point through the transcritical bifurcation and the disease approaches the endemic steady state $P_{1}$ on $F_{1}=0$. In case $R_{0}$ is equal to one, all the solutions in $\left(\mathbb{R}^{+}\right)^{3}$ tend to $P_{0}$ on $F_{1}=0$, which implies the disease is supposed to be controlled and the entire population tends to be healthy, but is susceptible to reinfection. These facts show that the basic reproduction number has played an important role in the spread of disease and the topological structure of orbits for the SIR model. The approach we used in this work may contribute to the understanding of the dynamics of the more general epidemic models.

\section{Data Availability}

All data included in this study are available upon request by contact with the corresponding author.

\section{Conflicts of Interest}

The authors declare that they have no conflicts of interest.

\section{Acknowledgments}

The author would like to acknowledge the financial support from the Scientific Research Program funded by Shaanxi Provincial Education Department (Program No. 19JZ051) and Xi'an Academy of Social Sciences (Program No. JG191).

\section{References}

[1] D. J. Daley and J. Gani, Epidemic Modeling: An Introduction, Cambridge University Press, Cambridge, 2005.

[2] W. O. Kermack and A. G. McKendrick, "A contribution to the mathematical theory of epidemics," Proceedings of the Royal Society of London. Series A, Containing Papers of a Mathematical and Physical Character, vol. 115, no. 772, pp. 700-721, 1997.

[3] H. S. Rodrigues and M. J. Fonseca, "Can information be spread as a virus? Viral marketing as epidemiological model," Mathematicsl Methods in the Applied Sciences, vol. 39, no. 16, pp. 4780-4786, 2016.

[4] M. Marin, R. Ellahi, and A. Chirilă, "On solutions of SaintVenant's problem for elastic dipolar bodies with voids," Carpathian Journal of Mathematics, vol. 33, no. 2, pp. 219-232, 2017.

[5] A. R. Seadawy, "Two-dimensional interaction of a shear flow with a free surface in a stratified fluid and its solitary-wave solutions via mathematical methods," The European Physical Journal Plus, vol. 132, no. 12, article 518, 2017.

[6] A. R. Seadawy and J. Manafian, "New soliton solution to the longitudinal wave equation in a magneto-electro-elastic circular rod," Results in Physics, vol. 8, pp. 1158-1167, 2018.

[7] A. R. Seadawy and K. El-Rashidy, "Dispersive solitary wave solutions of Kadomtsev-Petviashvili and modified Kadomtsev-Petviashvili dynamical equations in unmagnetized dust plasma," Results in Physics, vol. 8, pp. 1216-1222, 2018.

[8] A. R. Seadawy and S. Z. Alamri, "Mathematical methods via the nonlinear two-dimensional water waves of Olver 
dynamical equation and its exact solitary wave solutions," Results in Physics, vol. 8, pp. 286-291, 2018.

[9] A. H. Arnous, A. R. Seadawy, R. T. Alqahtani, and A. Biswas, "Optical solitons with complex Ginzburg-Landau equation by modified simple equation method," Optik, vol. 144, pp. 475-480, 2017.

[10] Abdullah, A. R. Seadawy, and W. Jun, "Mathematical methods and solitary wave solutions of three-dimensional ZakharovKuznetsov-Burgers equation in dusty plasma and its applications," Results in Physics, vol. 7, pp. 4269-4277, 2017.

[11] E. S. Selima, A. R. Seadawy, and X. Yao, “The nonlinear dispersive Davey-Stewartson system for surface waves propagation in shallow water and its stability," The European Physical Journal Plus, vol. 131, no. 12, article 425, 2016.

[12] A. R. Seadawy and K. El-Rashidy, "Nonlinear Rayleigh-Taylor instability of the cylindrical fluid flow with mass and heat transfer," Pramana, vol. 87, no. 2, 2016.

[13] A. R. Seadawy, "Solitary wave solutions of two-dimensional nonlinear Kadomtsev-Petviashvili dynamic equation in dustacoustic plasmas," Pramana, vol. 89, no. 3, 2017.

[14] G. Groza, M. Jianu, and N. Pop, "Infinitely differentiable functions represented into Newton interpolating series," Carpathian Journal of Mathematics, vol. 30, no. 3, pp. 309-316, 2014.

[15] C. Christopher, J. Llibre, and J. V. Pereira, "Multiplicity of invariant algebraic curves in polynomial vector fields," Pacific Journal of Mathematics, vol. 229, no. 1, pp. 63-117, 2007.

[16] X. Zhang, Integrability of Dynamical Systems: Algebra and Analysis, Springer, 2017.

[17] K. Huang, S. Shi, and W. Li, "Meromorphic and formal first integrals for the Lorenz system," Journal of Nonlinear Mathematical Physics, vol. 25, no. 1, pp. 106-121, 2018.

[18] K. Huang, S. Shi, and W. Li, "Integrability analysis of the Shimizu-Morioka system," Communications in Nonlinear Science and Numerical Simulation, vol. 84, article 105101, 2020.

[19] C. Valls, "Invariant algebraic surfaces and algebraic first integrals of the Maxwell-Bloch system," Journal of Geometry and Physics, vol. 146, article 103516, 2019.

[20] J. Llibre and C. Valls, "Integrability of reversible and equivariant quadratic polynomial differential systems in the plane," The Rocky Mountain Journal of Mathematics, vol. 49, no. 2, pp. 579-591, 2019.

[21] K. Huang, S. Shi, and W. Li, "First integrals of the MaxwellBloch system," Comptes Rendus Mathématique, vol. 358, no. 1, pp. 3-11, 2020.

[22] K. Huang, S. Shi, and Z. Xu, "Integrable deformations, biHamiltonian structures and nonintegrability of a generalized Rikitake system," International Journal of Geometric Methods in Modern Physics, vol. 16, no. 4, article 1950059, 2019.

[23] W. Szumiński and M. Przybylska, "Differential Galois integrability obstructions for nonlinear three-dimensional differential systems," Chaos, vol. 30, no. 1, article 013135, 2020.

[24] C. Valls, "Invariant algebraic surfaces for a virus dynamics," Zeitschrift für Angewandte Mathematik und Physik, vol. 66, no. 4, pp. 1315-1328, 2015.

[25] J. Llibre and X. Zhang, "Invariant algebraic surfaces of the Lorenz system," Journal of Mathematical Physics, vol. 43, no. 3, pp. 1622-1645, 2002.

[26] J. Llibre, R. Saghin, and X. Zhang, "On the analytic integrability of the 5-dimensional Lorenz system for the gravity-wave activity," Proceedings of the American Mathematical Society, vol. 142, no. 2, pp. 531-537, 2014.

[27] C. Valls, "Invariant algebraic surfaces for generalized Raychaudhuri equations," Communications in Mathematical Physics, vol. 308, no. 1, pp. 133-146, 2011.

[28] C. Valls, "On the global dynamics of the Newell-Whitehead system," Journal of Nonlinear Mathematical Physics, vol. 26, no. 4, pp. 569-578, 2019.

[29] J. Llibre, Y. Paulina Martínez, and C. Valls, "On the global dynamics of a three-dimensional forced-damped differential system," Journal of Nonlinear Mathematical Physics, vol. 27, no. 3, pp. 414-428, 2020.

[30] J. Biazar, "Solution of the epidemic model by Adomian decomposition method," Applied Mathematics and Computation, vol. 173, no. 2, pp. 1101-1106, 2006.

[31] H. Khan, R. N. Mohapatra, K. Vajravelu, and S. J. Liao, "The explicit series solution of SIR and SIS epidemic models," Applied Mathematics and Computation, vol. 215, no. 2, pp. 653-669, 2009.

[32] M. Rafei, H. Daniali, and D. D. Ganji, "Variational iteration method for solving the epidemic model and the prey and predator problem," Applied Mathematics and Computation, vol. 186, no. 2, pp. 1701-1709, 2007.

[33] M. Bohner, S. Streipert, and D. F. M. Torres, "Exact solution to a dynamic SIR model," Nonlinear Analysis: Hybrid Systems, vol. 32, pp. 228-238, 2019.

[34] H. T. Williams, I. Mazilu, and D. A. Mazilu, "Stochastic epidemic-type model with enhanced connectivity: exact solution," Journal of Statistical Mechanics: Theory and Experiment, vol. 2012, no. 1, article P01017, 2012.

[35] T. Harko, F. S. N. Lobo, and M. K. Mak, "Exact analytical solutions of the susceptible-infected-recovered (SIR) epidemic model and of the SIR model with equal death and birth rates," Applied Mathematics and Computation, vol. 236, pp. 184-194, 2014.

[36] A. Cima and J. Llibre, "Bounded polynomial vector fields," Transactions of the American Mathematical Society, vol. 318, no. 2, pp. 557-579, 1990.

[37] F. Dumortier, J. Llibre, and J. C. Artés, Qualitative Theory of Planar Differential Systems, Springer, 2006. 